\title{
NEGATIVE PRESSURE GENERATED BY OCTOPUS SUCKERS: A STUDY OF THE TENSILE STRENGTH OF WATER IN NATURE
}

\author{
BY ANDREW M. SMITH \\ Department of Biology, Coker Hall, CB 3280, The University of North Carolina, \\ Chapel Hill, NC 27559-3280, USA
}

Accepted 17 December 1990

\begin{abstract}
Summary
The decrease in hydrostatic pressure generated by octopus suckers adhering to wettable and non-wettable surfaces was measured using a flush-mounted miniature pressure transducer. The cavitation thresholds, or lowest sustainable pressures, of sea water on the same surfaces were also measured and were compared with the pressures generated by octopuses. It is shown that suckers can generate hydrostatic pressures below $0 \mathrm{MPa}$ on moderately wettable surfaces. This disproves the commonly repeated assumption that suckers cannot produce pressures below a vacuum and suggests that the importance of suction in attachment mechanisms may have been overlooked. On epoxy, the lowest recorded pressure was $-0.168 \mathrm{MPa}(0.268 \mathrm{MPa}$ or $2.66 \mathrm{~atm}$ below ambient), and the octopus generated negative pressure in $35 \%$ of the pulls that were considered maximal efforts. The suckers never generated negative pressures on non-wettable surfaces. These results are in agreement with the range of pressures that sea water can sustain on the same surfaces. It is suggested, therefore, that cavitation, the failure of water in tension, limits the attachment force of suckers. The difference between the cavitation threshold of water in nature and the cavitation threshold of pure water is discussed.
\end{abstract}

\section{Introduction}

Understanding suction attachment mechanisms requires knowledge of the physics of water under negative pressure (pressures below $0 \mathrm{MPa}$ ) (see Kier and Smith, 1990). If water is contained in a leak-proof, expandable container, such as a sucker, exerting force to expand the container decreases the pressure of the water with little actual increase in volume. This is because water's cohesiveness resists expansion, and the decrease in pressure balances the expansive force (see Denny, 1988; Kier and Smith, 1990). Water at decreased pressure behaves like a solid in tension because of its low expansibility (high bulk modulus). Water is thus sometimes described as having tensile strength. Water breaks when the pressure falls to the cavitation threshold for that particular sample. At the cavitation

Key words: Octopus suckers, cavitation, pressure, adhesion, water. 
threshold, microscopic gas bubbles suddenly grow without restraint in an event defined as cavitation (Pickard, 1981).

Previous analyses of attachment mechanisms in marine animals have been based, in part, on the assumption that suckers cannot generate pressures below $0 \mathrm{MPa}$. If one accepts the assumption that suckers are unable to generate pressures below $0 \mathrm{MPa}$, then the maximum pressure differential that a sucker could produce between the enclosed water and the water at ambient pressure outside the sucker is $0.1 \mathrm{MPa}(1 \mathrm{~atm})$ at sea level. This pressure differential produces a maximum tenacity, or attachment force per unit area, of nearly $0.1 \mathrm{MPa}$. Therefore, a sucker could not have greater than $0.1 \mathrm{MPa}$ tenacity without violating the assumption that suckers cannot generate negative pressures. Grenon and Walker (1981) and Branch and Marsh (1978) rule out a suction attachment mechanism on these grounds for limpets, as do Yule and Crisp (1983) for barnacle larvae.

Parker (1917, 1921), Paine (1926) and Nixon and Dilly (1977) also assumed that suckers have a maximum tenacity of $0.1 \mathrm{MPa}$ at sea level. Paine (1926) and Parker (1917) measured tenacities greater than $0.1 \mathrm{MPa}$ from starfish tube feet and sea anemone suckers, respectively, but attributed the excess tenacity to the use of glue or to experimental error because they assumed that suction could only account for $0.1 \mathrm{MPa}$ of the tenacity.

The assumption that suckers cannot generate negative pressures has not been tested. In fact, water can withstand negative pressures in laboratory experiments (Hayward, 1971; Pickard, 1981). Denny (1988) and Kier and Smith (1990) suggest that water is also able to sustain negative pressures in suckers. Research into the mechanism of sap ascent in vascular plants has demonstrated water's ability to sustain negative pressures. According to the evaporation transport model of sap ascent, vascular plants pull water up from their roots to their leaves. This means that the water in the xylem elements is in tension, and the pressure in certain species may drop to $-8 \mathrm{MPa}$ (Scholander et al. 1965).

Negative pressures in water are clearly possible, but can suckers generate negative pressure and, if so, what magnitude of pressures do they generate? Does the cavitation threshold of water or the mechanics of the sucker muscles limit a sucker's tenacity? This study was designed to answer these questions. Most published measurements of cavitation threshold are for pure samples of distilled water rather than for samples of water from nature. I therefore determined the cavitation threshold of sea water and used this to predict the pressure that octopuses could generate. I then used a miniature pressure transducer to measure the pressures generated by octopus suckers.

\section{Materials and methods}

\section{Cavitation threshold of sea water}

A Z-tube apparatus based on Briggs's (1950) design was built to measure the cavitation threshold of sea water (Fig. 1). A $373 \mathrm{~W}$ (0.5 h.p.) variable-speed electric motor with remote speed control was mounted on a plywood frame so that 
the axis of rotation was vertical. Glass tubes $(3 \mathrm{~mm}$ internal diameter) were bent into a Z-shape and glued with Devcon 5 min epoxy gel to individual $30 \mathrm{~cm} \times 7 \mathrm{~cm} \times$ $0.3 \mathrm{~cm}$ Lucite (acrylic) sheets. Each sheet, with its Z-tube, could be bolted to a $30 \mathrm{~cm}$ diameter aluminum disk mounted on the motor shaft. Tubes were slowly filled with water, taking care that there were no visible air bubbles. Then the disk and attached tube were spun at gradually increasing speeds.

Centrifugal force pulled the water towards the ends of the tube, generating tension. The highest tension (and thus lowest pressure) was at the center of rotation. The Z-shape prevented water from spraying out of the ends of the tube by balancing exactly equal amounts of water on either side of the tube (Fig. 1B).

The entire apparatus was encased in a heavy pine box with a clear Lucite top. The box reduced the chance of personal injury as rotational velocities up to 4000 revs $\mathrm{min}^{-1}$ were used and tubes occasionally broke loose. The apparatus
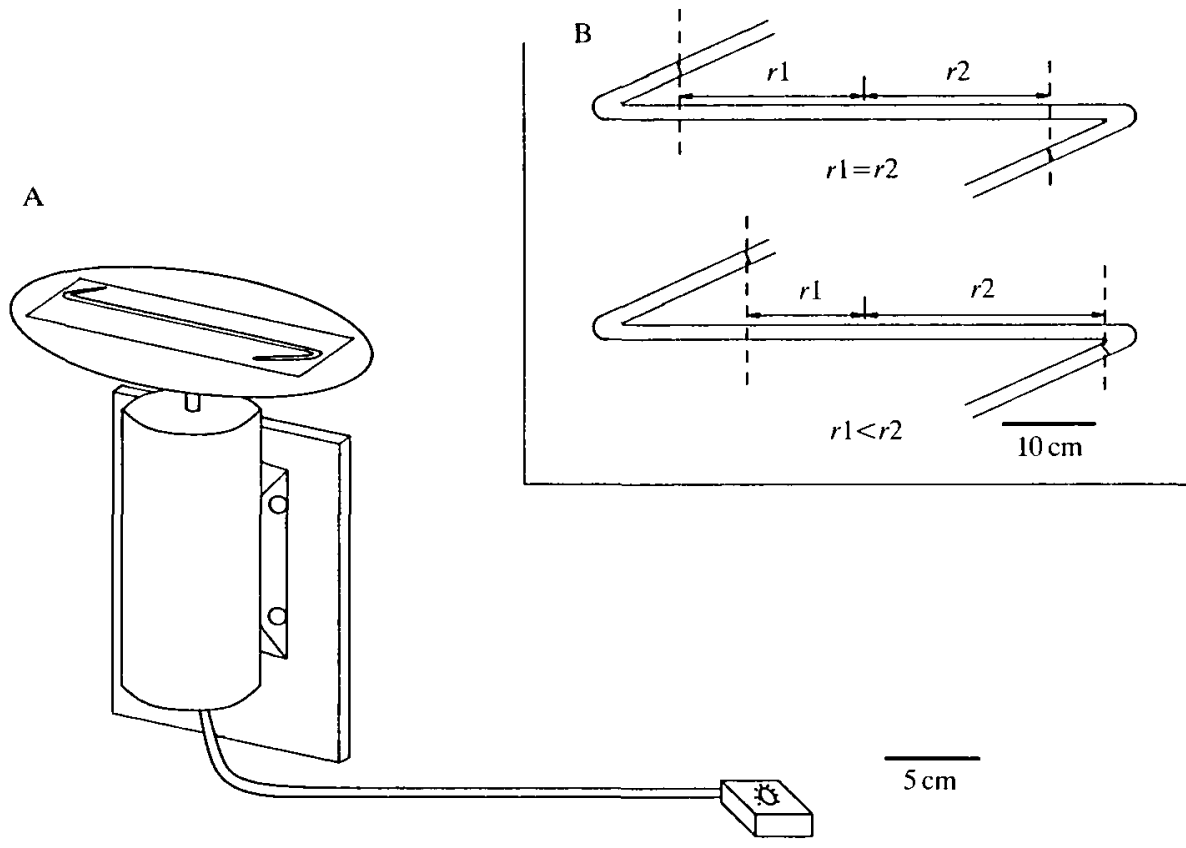

Fig. 1. (A) Schematic diagram of the Z-tube apparatus used to measure the cavitation threshold of sea water. (B) The Z-tube balances the water so that it does not spray out at the ends. The water in the arm of the $Z$ will be pushed away from the tube opening by centrifugal force and will cancel the force exerted by the water the same distance from the center in the main part of the tube. Thus, the net force pulling away from the center comes from the volume of water shown as $r 1$ and $r 2$. The tube is in equilibrium when $r 1=r 2$. If, for example, water were to flow towards the left, $r 1$ would become shorter, because there would be more water in the left-hand arm cancelling the force from a larger portion of the main tube, and $r 2$ would become longer, because there would be less water in that arm of the $Z$. Thus, the water in the right half will exert a larger net force away from the center and will pull water back over to its side, reestablishing equilibrium. 
differed from Briggs's in that it was not enclosed in a vacuum chamber. Performing the tests in a vacuum removes dissolved gases from the water, which significantly lowers the cavitation threshold, increasing the magnitude of the pressure drop (Greenspan and Tschiegg, 1967). Since water in nature contains dissolved gases, a vacuum chamber was not used.

Cavitation was observed with a variable-speed stroboscope (1546 Strobotac, GenRad, Inc., Concord, MA). Synchronizing the frequency of the stroboscope with the rotation of the disk gives the appearance of freezing the motion of the disk so that cavitation could be observed and the exact rotational velocity of the disk at that point recorded. The pressure was calculated from the rotational velocity of the disk and the distance of the meniscus from the center of the tube ( $r 1$ or $r 2$ in Fig. 1B). The equation is derived by integrating the equation for centrifugal force, $F=m w^{2} r$, over the length of the tube, resulting in, $P=0.5 \sigma w^{2} r^{2}$, where $P$ is the pressure, $\sigma$ is the density of the fluid, $w$ is the angular velocity and $r$ is the distance from the center of the tube to the meniscus.

The accuracy of the Z-tube was checked by introducing tiny gas bubbles into the water sample to be tested. These bubbles are small enough, so that, when they expand to the width of the tube's lumen, we can assume that the pressure inside the bubble, and thus in the water at the center, must be approximately $0 \mathrm{MPa}$. The expansion of the bubble occurs abruptly and is not difficult to detect. The pressure was measured when the bubbles reached this point and was found to be $0.008 \pm 0.006 \mathrm{MPa}$ (mean \pm s.D., $N=101$ ). This was rechecked throughout the experiments and found to be consistent.

The cavitation threshold of sea water and deionized, distilled water was measured in Z-tubes lined with one of several different surfaces. The Z-tubes were either cleaned with a $2 \%$ solution of potassium dichromate in $10 \%$ sulfuric acid (Humason, 1979, p. 571), or completely lined with a thin layer of wax, silicone grease or Devcon 2-ton epoxy adhesive. The tubes were lined by pouring molten wax through them, or by using a monofilament fishing line to drag a small piece of clean laboratory wiper (Kimwipe) soaked in silicone grease or unpolymerized epoxy through the lumen at least five times. The coating did not decrease the diameter of the lumen significantly. A large sample of sea water was taken from the ocean at a pier near Morehead City, NC, and kept refrigerated. Samples of Instant Ocean artificial sea water (Aquarium Systems, Mentor, OH) were taken directly from a tank containing octopuses. Samples of distilled water had passed through a submicron filter, an activated carbon filter and two successive deionizing resin cartridges, resulting in water of at least $18 \mathrm{M} \Omega$ purity (Hydro service and supplies, Research Triangle Park, NC). More than 50 samples of water were tested over the course of 2 years. In most cases, 10 tests were run on each sample.

\section{Pressure measurement under octopus suckers}

Specimens of Octopus vulgaris Cuvier were supplied by the Duke University Marine Laboratory (Beaufort, NC). Specimens of the Octopus bimaculoides/ bimaculatus complex (see Pickford and McConnaughey, 1949) were supplied by 
Winkler Enterprises (San Pedro, CA). Octopuses were kept at approximately $20^{\circ}$ $\mathrm{C}$ in a closed artificial seawater aquarium (see Forsythe and Hanlon, 1980; Hanlon and Forsythe, 1985). Animals lived in one tank, water was pumped from this tank to a separate conditioning tank and then through a system of filters before returning to the main tank. The filters included a mechanical filter, an activated carbon filter and an ultraviolet sterilization module (Rainbow Plastics, El Monte, CA).

The pressure generated by octopus suckers was measured with a $1.5 \mathrm{~mm}$ diameter pressure transducer (model 060s, Precision Measurements Co., Ann Arbor, MI). The transducer was glued into a shallow depression near the tip of a hollow Lucite rod using Devcon 2-ton epoxy so that the sensing face was flush with the outer surface (Fig. 2). The rod was made by gluing four $30 \mathrm{~cm} \times 3 \mathrm{~cm}$ Lucite strips together, creating a hollow space in the center for the wiring. The ends of the rod were sealed to protect the wiring from sea water. The wires were passed inside the rod under waterproof coatings, and exited the rod through Tygon tubing. The part of the rod exposed to the suckers was coated with either epoxy or silicone grease so that there was a uniform surface free of macroscopic defects.

The transducer formed one arm of a Wheatstone bridge, and its output was amplified and sent to a Gould Electronics chart recorder. The transducer was calibrated by placing the entire sensing apparatus in a container of water inside a vacuum chamber and reducing the pressure to values ranging from 0.040 to 0.005 $\mathrm{MPa}$ as measured by a Bourdon vacuum gauge. The regression equation of the calibration curve predicts pressures that have an average $5 \%$ error using the reading of the vacuum gauge as a standard. Because the transducer is flush with the surface, the error will not increase significantly as the rate of pressure change increases (Gabe, 1972). Care was taken to eliminate drift due to resistive heating

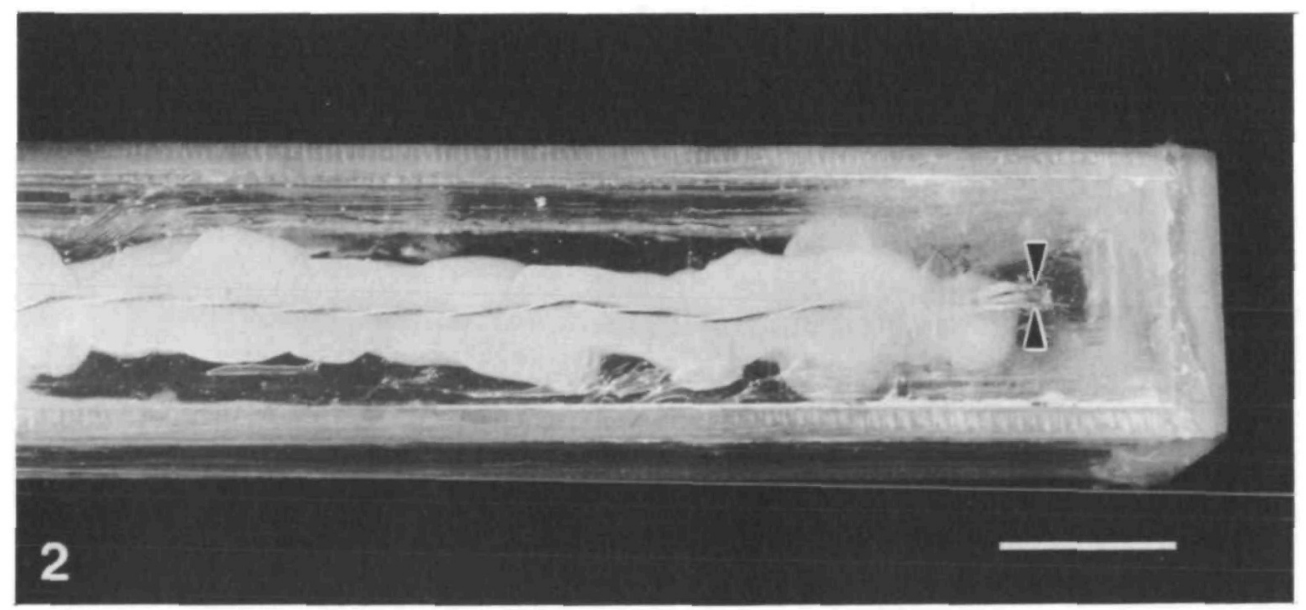

Fig. 2. The apparatus used to measure the pressure under octopus suckers. The miniature pressure transducer is embedded near the tip of the Lucite rod, between the arrowheads. Scale bar, $20 \mathrm{~mm}$. 
of the transducer. This was initially a problem because of the small size of the transducer, but it was eliminated by decreasing the excitation voltage to $2 \mathrm{~V}$.

After turning off the airstones in the aquarium, the pressure-sensing apparatus was presented to the octopus. The octopuses were trained with food rewards to grab the rod. When a sucker was observed to attach over the transducer, the rod was pulled away slowly but firmly so that the octopus would grip it with maximal force. Nevertheless, it was not possible to ensure that each pull represented a maximal effort. Also, the suckers were not always centered over the transducer and sometimes pressed on its edge. The resulting positive pressure would cancel some of the decrease in pressure, resulting in lower output from the transducer. Thus, on average, the pressures measured are probably a slight underestimate of the maximum pressures possible.

The pressures that were clearly not due to maximal pulls were excluded from the analysis. These included all of the pressures from $0.1 \mathrm{MPa}$ (ambient) down to $0.02 \mathrm{MPa}$. Octopus suckers frequently generated pressures lower than this, so the strength of the muscle is not limiting in this range. Also water's tensile strength is not limiting in this range. Pulls that generate pressures above $0.02 \mathrm{MPa}$ cannot be limited by cavitation because the highest pressure at which cavitation occurs is $0.008 \pm 0.006 \mathrm{MPa}(N=101)$, based on the calibration of the Z-tube. For pressures above $0.02 \mathrm{MPa}$, newly formed air bubbles will not even expand to become visible, and there is no reason for the sucker's attachment to fail unless a leak forms or the animal lets go. Therefore, these pressures were not considered in the comparison with the data from the Z-tube.

Non-parametric statistics were used for all comparisons because the data were not normally distributed.

\section{Results \\ Z-tube}

Sea water can sustain negative pressures (Table 1). However, the cavitation thresholds of sea water are not as low as values previously reported for pure water, and there is considerable variability in the measurements on each surface (Table 1, Fig. 3). Distilled water also showed a broad distribution of cavitation thresholds.

Table 1. Mean cavitation thresholds of water on different surfaces

\begin{tabular}{|c|c|c|c|c|c|c|c|}
\hline & \multicolumn{3}{|c|}{ Glass } & \multicolumn{2}{|c|}{ Epoxy } & \multicolumn{2}{|c|}{ Non-wettable } \\
\hline & OSW & ASW & DW & ASW & DW & ASW & DW \\
\hline$P(\mathrm{MPa})$ & -0.056 & -0.073 & -0.258 & -0.061 & -0.199 & 0.009 & -0.034 \\
\hline S.D. & 0.106 & 0.153 & 0.327 & 0.147 & 0.108 & 0.006 & 0.075 \\
\hline$N$ & 60 & 116 & 192 & 127 & 22 & 30 & 26 \\
\hline
\end{tabular}

OSW, ocean sea water; ASW, artificial sea water; DW, distilled water; $P$, pressure. 


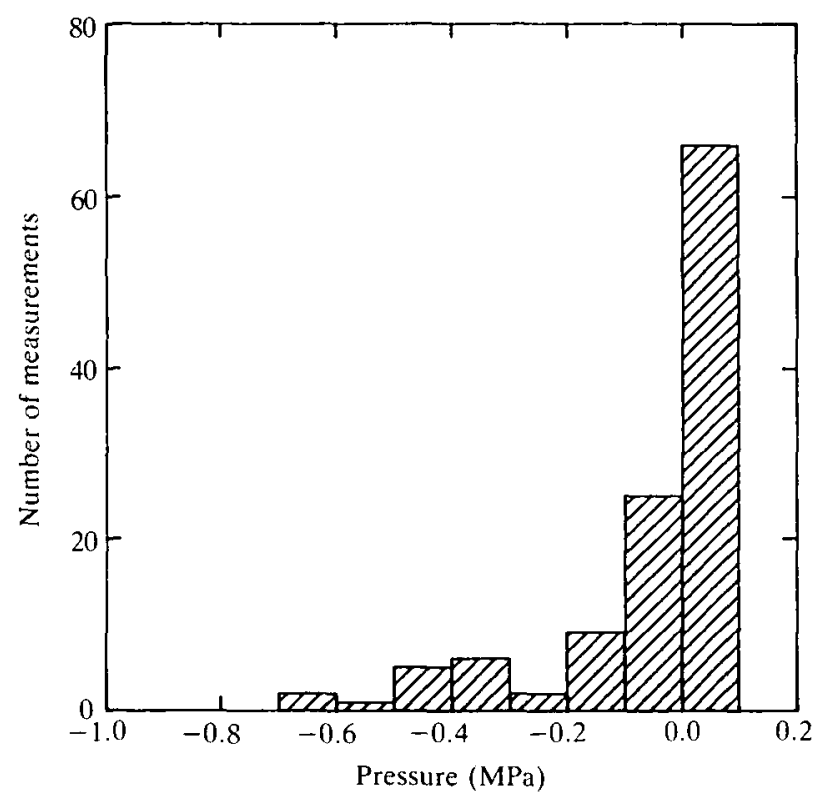

Fig. 3. Distribution of cavitation thresholds measured for artificial sea water in a cleaned glass Z-tube, $N=116$. The variability shown here is typical for cavitation threshold measurements.

In $16 \%$ of the tests, distilled water on glass sustained pressures below $-0.7 \mathrm{MPa}$, which is the range expected from published values (Couzens and Trevena, 1969; Greenspan and Tschiegg, 1967; Trevena, 1967; Wilson et al. 1975; Sedgewick and Trevena, 1976), but $29 \%$ of the time cavitation occurred between 0.015 and $0 \mathrm{MPa}$.

The mean cavitation thresholds of ocean sea water and aquarium sea water are not significantly different (Wilcoxon two-sample test, $P=0.75$ on glass), but aquarium sea water is weaker than distilled water (Wilcoxon two-sample test, $P=0.0001$ on glass and on epoxy, $P=0.024$ on non-wettable surfaces). There was some variability among samples taken from the same source at different times, but the differences among the mean cavitation thresholds of different samples were significant for only one of four tubes in which different samples were tested (Kruskal-Wallis test, $P=0.004, P=0.09, P=0.41, P=0.14$ ). The one tube in which there were statistically significant differences among samples had six or fewer trials for seven of the nine samples, which makes its significance questionable.

The characteristics of the wetted surface affect water's cavitation threshold (Table 1). Wettability, or surface energy, is particularly important in this context. A useful empirical measure of wettability is $\tau_{c}$, the critical surface tension. Wettable surfaces have higher values of $\tau_{\mathrm{c}}$ than non-wettable surfaces (see Baier et al. 1968). $\tau_{\mathrm{c}}$ of glass is $45 \times 10^{-5} \mathrm{~N} \mathrm{~cm}^{-1}$ (Baier, 1970). $\tau_{\mathrm{c}}$ of Epon, which should be similar to the epoxy used here, is $37 \times 10^{-5} \mathrm{~N} \mathrm{~cm}^{-1}$, and $\tau_{\mathrm{c}}$ of silicone is 

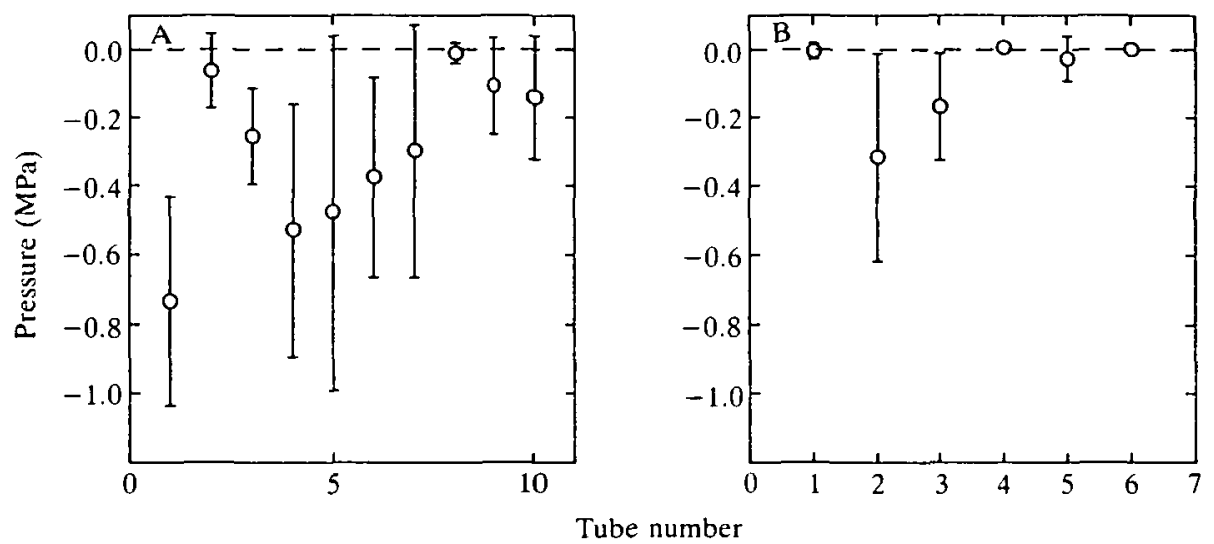

Fig. 4. Mean cavitation thresholds measured in different tubes. (A) Deionized distilled water tested in 10 different clean glass tubes. A Kruskal-Wallis test showed that the differences among the mean cavitation thresholds measured in different tubes were statistically significant $(P=0.0001, N=184)$. (B) Artificial sea water tested in six different epoxy-lined $\mathrm{Z}$-tubes. The differences among mean cavitation thresholds measured in different tubes were statistically significant $(P=0.0001, N=127)$. The error bars represent standard deviations.

$22 \times 10^{-5} \mathrm{~N} \mathrm{~cm}^{-1}$ (Shafrin, 1975). The difference between cavitation thresholds on glass and on epoxy is not statistically significant (Wilcoxon two-sample test, $P=0.48$ for aquarium sea water, $P=0.25$ for distilled water), but non-wettable surfaces affect the cavitation thresholds dramatically. Cavitation thresholds are lower (more negative) on glass or epoxy than on wax or silicone grease (Wilcoxon two-sample test, $P=0.0001$ for aquarium sea water, $P=0.0001$ for distilled water). In 30 trials on non-wettable surfaces, aquarium sea water always cavitated at or slightly above $0 \mathrm{MPa}$. On these surfaces, cavitation occurred simultaneously at 3-10 individual nucleating sites, whereas on more wettable surfaces, such as epoxy or glass, only one cavitation event was typically observed.

The cavitation threshold's dependence on surfaces was also apparent in the variability among tubes. Using cleaned glass tubes filled with deionized, distilled water, which was extremely constant in quality, it was predicted that there would be no differences among cavitation thresholds measured in different tubes. This hypothesis was rejected (Kruskal-Wallis test, $P=0.0001$ ). There are statistically significant differences among the cavitation thresholds measured in different glass tubes (Fig. 4A). The same was true of epoxy-lined tubes filled with artificial sea water (Kruskal-Wallis test, $P=0.0001$; Fig. $4 \mathrm{~B}$ ). With some tubes, it was clear that cavitation almost always occurred at approximately $0 \mathrm{MPa}$, often at several different nucleating sites. Yet water from the same sample, tested in a different tube with the same type of surface, consistently sustained negative pressures.

\section{Octopus pressure measurements}

In both octopus species tested, suckers on an epoxy surface can generate 


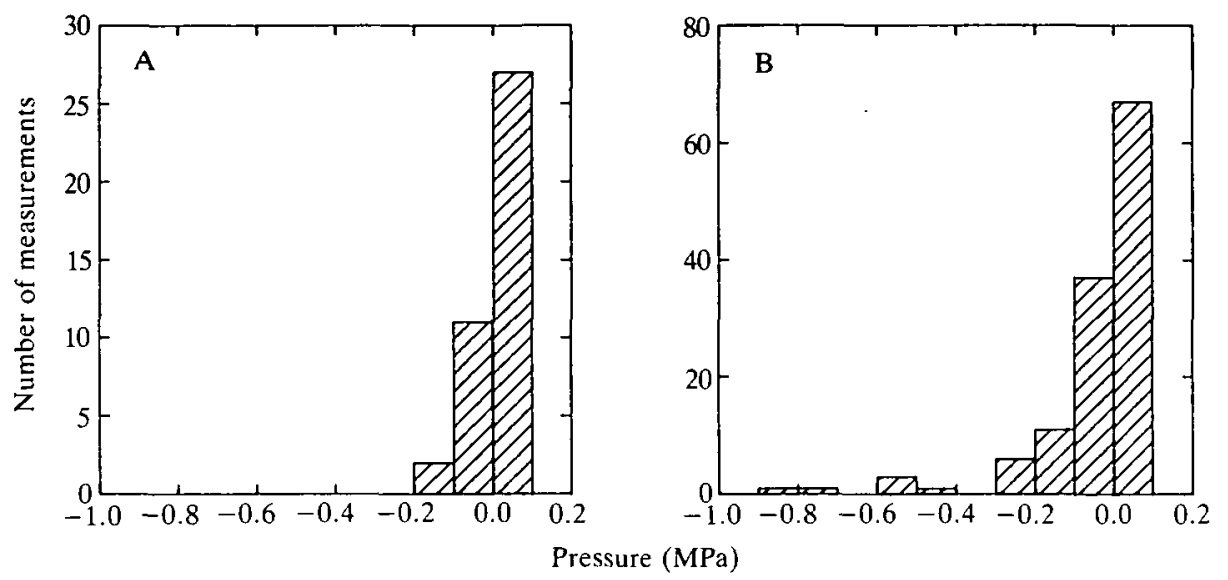

Fig. 5. (A) The distribution of pressures measured under octopus suckers attached to epoxy, excluding pressures above $0.02 \mathrm{MPa}, N=40$. (B) The distribution of cavitation thresholds of artificial sea water in epoxy-lined Z-tubes, $N=127$.

negative pressure (Fig. 5A). For comparison, the distribution of cavitation thresholds measured in epoxy-lined Z-tubes is shown (Fig. 5B). There is no statistically significant difference between these two distributions (KolmogorovSmirnov test for goodness of fit, $P>0.2$ ). The lowest pressure recorded under an octopus sucker was $-0.168 \mathrm{MPa}$ (2.66 atm below ambient). Out of 140 trials where any pressure drop was recorded, 100 were pressures above $0.02 \mathrm{MPa}$ and were thus not considered maximal. These often occurred when the octopus gently explored the rod. Of the 40 considered maximal, 14 were negative and 26 were between 0 and $0.02 \mathrm{MPa}$. The octopuses were able to generate negative pressures not only when the rod was pulled away, but also when they held and manipulated it. Also, on many of the hard pulls numerous popping sounds could be heard and felt through the rod as it was pulled away from the octopus. In several cases, small bubbles were observed to rise from the animal's web from the location where it had held the rod. These bubbles may represent gas produced by cavitation in the sucker.

After coating the pressure transducer with silicone grease, in 117 trials where a sub-ambient pressure registered, the octopus never generated negative pressures. This is significantly different from the distribution of pressures generated by octopuses on epoxy (Kolmogorov-Smirnov test for goodness of fit, $P<0.0001$ ).

\section{Discussion}

\section{Negative pressures and sucker tenacity}

Because suckers can generate negative pressures, there is no pre-established limit on their tenacity. Although sea water often cavitates at $0 \mathrm{MPa}$, it frequently sustains much lower pressures. This means that suction cannot automatically be 
ruled out in attachment mechanisms that achieve tenacities greater than $0.1 \mathrm{MPa}$ at sea level. In the light of these results, previous studies of attachment should be reviewed to see if the role of suction has been overlooked or underestimated.

Paine (1926) found tenacities greater than $0.1 \mathrm{MPa}$ for starfish tube feet. She attributed the excess tenacity to the presence of a glue and supported this by creating a leak under the adhesive disk and measuring the remaining tenacity, which accounted for all the tenacity in excess of $0.1 \mathrm{MPa}$. Additional evidence has supported the hypothesis that tube feet use a glue (Hermans, 1983; Thomas and Hermans, 1985).

Similarly, Grenon and Walker (1981) found that limpet tenacities exceeded $0.1 \mathrm{MPa}$ and suggested that limpet attachment was based on the viscoelastic properties of mucus. In the case of limpet attachment, however, it has been found that suction plays a major role (A. M. Smith, in preparation).

Parker (1917) found the mean tenacity of sea anemone (Cribrina) suckers to be approximately $0.1 \mathrm{MPa}$. Thus, he assumed that the suckers had reached a physical limit determined by a vacuum. Nevertheless, some of the tenacity measurements were greater than $0.1 \mathrm{MPa}$. Seven of ten measurements fell between 0.077 and $0.106 \mathrm{MPa}$, but the other three were between 0.136 and $0.162 \mathrm{MPa}$. These tenacities correspond to a distribution of pressures similar to that generated by octopus suckers on an epoxy surface.

Emerson and Diehl (1980), Stork (1980) and Lees and Hardie (1988) tested for suction adhesion in tree frogs, beetles and aphids, respectively, by putting the animals in a pressure chamber and lowering ambient pressure. This would eliminate much of the pressure differential, and thus the attachment force, of a sucker that could not generate pressures below $0 \mathrm{MPa}$. Because in all three cases the tenacity of these animals was maintained, the authors concluded that a suction mechanism was not used. Nevertheless, these experiments do not disprove the hypothesis that these animals use suction, because the animals produce secretions between their feet and the substratum that could presumably sustain pressures below $0 \mathrm{MPa}$, and thus could maintain a pressure differential in a vacuum.

In support of a $0.1 \mathrm{MPa}$ limit on sucker tenacity, Parker (1921) found that the tenacity of octopus suckers was less than $0.1 \mathrm{MPa}$. However, it is likely that he performed the tests in air without ensuring that the suckers were water-filled. The maximum tenacity at sea level of an air-filled sucker is $0.1 \mathrm{MPa}$. This is because, at best, such a sucker can only create a vacuum. In order to measure negative pressures, it is important always to keep the sensing apparatus under water to avoid inclusion of air pockets under the sucker. Even under water, introducing measuring devices under the sucker is likely to cause cavitation because water under negative pressures is metastable, like superheated liquids.

Since suckers can generate negative pressures, what determines the maximum tenacity of a sucker? The pressure, and thus tenacity, generated by a sucker is determined by the cavitation threshold of water, by the mechanics of the suction cup and its ability to avoid leaks or by the maximum stress that the sucker musculature generates. During maximal pulls, cavitation appears to limit the 
tenacity. The pressure transducer experiments show that octopus suckers generate the range and distribution of pressures predicted by the Z-tube for sea water on each surface. The octopuses did not generate negative pressures on non-wettable surfaces, as predicted from the Z-tube experiments. On epoxy, the distribution of pressures generated by octopus suckers was not significantly different from that predicted from the epoxy-lined Z-tubes, despite the variability between Z-tubes.

\section{The cavitation threshold of water in nature}

Although sea water can sustain negative pressures, the cavitation threshold measured in this study is higher (less negative) than that predicted by most previous work. Studies of the mechanism of sap ascent showed that the water in the xylem elements of a variety of hardwoods failed in tension at pressures ranging from -1 to $-5 \mathrm{MPa}$ (Milburn and McLaughlin, 1974; Pena and Grace, 1986; Sperry, 1986; Tyree and Dixon, 1986). Published values of water's cavitation threshold measured in the laboratory typically range from $-0.7 \mathrm{MPa}$ to $-2 \mathrm{MPa}$ (Couzens and Trevena, 1969; Greenspan and Tschiegg, 1967; Wilson et al. 1975; Sedgewick and Trevena, 1976), although Briggs (1950) and Trevena (1967) measured pressures as low as $-27.4 \mathrm{MPa}$ and $-5 \mathrm{MPa}$, respectively. There is, however, no mention of sample size or variance in the laboratory studies.

The higher cavitation threshold of sea water reported here and the broad range of published values may be explained by the presence of impurities. Particles suspended in water and defects on the wetted surface probably limit the cavitation threshold. These provide nucleating sites that stabilize gas bubbles, which then grow when the pressure drops to the threshold level, initiating cavitation (see Pickard, 1981). Boiling chips work on this same principle, although boiling is driven by an increase in temperature rather than a decrease in pressure. Thus, the presence and nature of the weakest link determines the cavitation threshold of a given sample of water. This weak link corresponds to the most likely nucleating site, usually a hydrophobic crack (Trevena, 1967; Pickard, 1981). Messinó et al. $(1963,1967)$ emphasize the variability in cavitation thresholds and point out that the cavitation threshold of water is only a measurement of the dimensions and wettability of the available nucleating sites for cavitation. This explains why sea water cavitates at a higher pressure than distilled water, because it has many more suspended particles and thus potential nucleating sites. Greenspan and Tschiegg (1967) mention that, if water is not purified by removing suspended particles, its cavitation threshold is variable and cavitation often occurs at $0 \mathrm{MPa}$, which is in agreement with the results of this study.

Because of the variability in water's cavitation threshold, it is probably not safe to apply values from the literature to situations in nature unless the surfaces and the presence of suspended impurities are strictly comparable. These factors can cause variation over several orders of magnitude. Briggs's (1950) Z-tube studies, which reported pressures as low as $-27.4 \mathrm{MPa}$, have been widely quoted by biologists, despite the fact that such pressures are much lower than have been achieved in other laboratory experiments. Briggs tested degassed, ultra-filtered, 
distilled water in a vacuum. His results are probably not applicable to biological systems.

The importance of nucleating sites may explain the variability between tubes in this study. Tubes 2 and 3 in Fig. 4B sustained significantly lower pressures than the other epoxy-lined tubes, probably because their surface was different in a way that is not clear at present, but that could involve a lack of minute impurities or surface defects.

\section{The cavitation threshold of water in trees}

Trees are able to put water under lower pressures than octopus suckers. This may be because they filter water across their roots, and because the xylem walls are wettable (Oertli, 1971; Pickard, 1981). Nevertheless, embolisms form in xylem elements (Milburn, 1973a ,b; Milburn and McLaughlin, 1974; Salleo and LoGullo, 1986). Because of theoretical estimates of water's cavitation threshold, which range down to $-200 \mathrm{MPa}$ (Pickard, 1981), and the previously mentioned published values of pure water's cavitation threshold, some researchers have questioned whether cavitation can even occur in xylem (Pickard, 1981, 1989), and others have proposed alternatives to cavitation as a means of embolism formation (Zimmerman, 1983; Crombie et al. 1985; Lewis, 1988; Sperry and Tyree, 1988). Nevertheless, we cannot rule out cavitation, because the tensile strength of xylem sap on surfaces comparable to xylem walls has never been tested and the potential cavitation thresholds range from $0 \mathrm{MPa}$ to $-27 \mathrm{MPa}$.

\section{How water's cavitation threshold affects suction adhesion}

Given an understanding of water under negative pressures, there are several interesting factors affecting the tenacity of an animal that uses suction. These include the variability in cavitation thresholds, the depth dependence of tenacity and the importance of the wettability of the surface.

The variability in water's tensile strength affects animals that use suckers. A sucker can form a strong attachment if it generates negative pressure, but the pressure that the water can withstand may not be predictable. This might provide an advantage to an animal that uses many suckers, such as an octopus, because it does not depend on only one sample of water.

Several authors have discussed the effect of depth on the tenacity of suckers (Nixon and Dilly, 1977; Able and McAllister, 1980; Kier and Smith, 1990). If sea water had a cavitation threshold of $-0.1 \mathrm{MPa}$, then at sea level $(0.1 \mathrm{MPa}$ ambient pressure) the sucker could generate $0.2 \mathrm{MPa}$ pressure differential from ambient before cavitation occurred. The same sucker at a depth of $90 \mathrm{~m}$ (1.0 MPa ambient pressure) could generate a $1.1 \mathrm{MPa}$ pressure differential before cavitation occurred, assuming that the sucker avoided leaks and that the sucker musculature could generate the required force. Thus, a small sucker in deep water could generate the same force as a large sucker in shallow water (Kier and Smith, 1990). The effect of depth on the tenacity of suckers may explain why shallow-water octopuses generally have larger suckers than deep-water octopuses (Voight, 1990). 
The dependence of the cavitation threshold on surface characteristics is an important consideration for future work. Crum (1979) found that decreasing surface tension decreased a liquid's cavitation threshold, which suggests that, if a surface is more easily wetted, the cavitation threshold will be lower. In this study, octopuses never generated negative pressures on non-wettable surfaces. Negative pressures were generated on epoxy, which is moderately wettable. A more quantitative comparison of pressures and wettability was not possible owing to the wide variation in the quality of similar surfaces as shown by the variation between individual Z-tubes. Further research is required to investigate the effect of surface characteristics on the cavitation threshold and to see if more-wettable surfaces allow lower pressures.

I would like to thank W. M. Kier for thoughtful discussions and technical help throughout this research. I am grateful to W. M. Kier and anonymous reviewers for comments on the manuscript. The Marine Biomedical Institute of the University of Texas Medical Branch at Galveston provided assistance with animal maintenance and supply. J. D. Robertson and co-workers were helpful in providing specimens of $O$. vulgaris. I thank S. Vogel and W. E. Bollenbacher for the use of their electronic equipment. This material is based upon work supported by a National Science Foundation Graduate Fellowship, a grant-in-aid of research from Sigma-Xi and a National Science Foundation Presidential Young Investigator Award (DCB-8658069) to W. M. Kier.

\section{References}

Able, K. W. And McAllister, D. E. (1980). Revision of the snailfish genus Liparis from arctic Canada. Can. Bull. Fish. aquat. Sci. 208, 1-58.

BAIER, R. E. (1970). Surface properties influencing biological adhesion. In Adhesion in Biological Systems (ed. R. S. Manly), pp. 15-48. New York: Academic Press.

Baier, R. E., Shafrin, E. G. and Zisman, W. A. (1968). Adhesion: mechanisms that assist or impede it. Science 162, 1360-1368.

Branch, G. M. And Marsh, A. C. (1978). Tenacity and shell shape in six Patella species: adaptive features. J. exp. mar. Biol. Ecol. 34, 111-130.

Briggs, L. J. (1950). Limiting negative pressure of water. J. appl. Phys. 21, 721-722.

Couzens, D. C. F. and Trevena, D. H. (1969). Critical tension in a liquid under dynamic conditions of stressing. Nature 222, 473-474.

Crombie, D. S., Hipkins, M. F. And Milburn, J. A. (1985). Gas penetration of pit membranes in the xylem of Rhododendron as the cause of acoustically detectable sap cavitation. Aust. $J$. Plant Physiol. 12, 445-453.

Crum, L. A. (1979). Tensile strength of water. Nature 278, 148-149.

Denny, M. (1988). Biology and the Mechanics of the Wave-swept Environment. Princeton: Princeton University Press.

Emerson, S. B. AND DieHL, D. (1980). Toe pad morphology and mechanisms of sticking in frogs. J. Linn. Soc. Biol. 13, 199-216.

Forsythe, J. W. AND HANLON, R. T. (1980). A closed marine culture system for rearing Octopus joubini and other large egged benthic octopods. Lab. Anim. 14, 137-142.

GABE, I. T. (1972). Pressure measurement in experimental physiology. In Cardiovascular Fluid Dynamics (ed. D. H. Bergel), pp. 11-50. London: Academic Press.

GREENSPAN, M. AND TSCHIEGG, C. E. (1967). Radiation-induced acoustic cavitation; apparatus and some results. J. Res. nat. Bur. Stand. 71C, 299-312. 
Grenon, J. F. and Walker, G. (1981). The tenacity of the limpet, Patella vulgata L.: an experimental approach. J. exp. mar. Biol. Ecol. 54, 277-308.

Hanlon, R. T. AND Forsythe, J. W. (1985). Advances in the laboratory culture of octopuses for biomedical research. Lab. Anim. 35, 33-40.

HaYward, A. T. J. (1971). Negative pressure in liquids: Can it be harnessed to serve man? Am. Sci. 59, 434-443.

Hermans, C. O. (1983). The duo-gland adhesive system. Oceanogr. mar. Biol. A. Rev. 21, 283-339.

Humason, G. L. (1979). Animal Tissue Techniques. San Francisco: W. H. Freeman and Co.

KIER, W. M. AND SMITH, A. M. (1990). The morphology and mechanics of octopus suckers. Biol. Bull. mar. biol. Lab., Woods Hole 178, 126-136.

LeEs, A. D. AND HaRdie, J. (1988). The organs of adhesion in the aphid Megoura viciae. J. exp. Biol. 136, 209-228.

LEwIS, A. M. (1988). A test of the air-seeding hypothesis using Sphagnum hyalocysts. Plant Physiol. 87, 577-582.

Messino, D., Sette, D. and Wanderlingh, F. (1963). Statistical approach to ultrasonic cavitation. J. acoust. Soc. Am. 35, 1575-1583.

Messin6, D., Sette, D. and Wanderlingh, F. (1967). Effects of solid impurities on cavitation nuclei in water. J. acoust. Soc. Am. 41, 573-583.

Milburn, J. A. (1973a). Cavitation in Ricinus by acoustic detection: induction in excised leaves by various factors. Planta 110, 253-265.

Milburn, J. A. (1973b). Cavitation studies on whole Ricinus plants by acoustic detection. Planta 112, 333-342.

Milburn, J. A. and Mclaughlin, M. E. (1974). Studies of cavitation in isolated vascular bundles and whole leaves of Plantago major L. New Phytol. 73, 861-871.

Nixon, M. ANd Dilly, P. N. (1977). Sucker surfaces and prey capture. Symp. zool. Soc. Lond. 38, 447-511.

Oertli, J. J. (1971). The stability of water under tension in the xylem. Z. Planzenphysiol. 65, 195-205.

Paine, V. (1926). Adhesion of the tube feet in starfishes. J. exp. Zool. 45, 361-366.

PARKer, G. H. (1917). The power of suction in the sea anemone Cribrina. J. exp. Zool. 24, 219-222.

Parker, G. H. (1921). The power of adhesion in the suckers of Octopus bimaculatus Verril. J. exp. Zool. 33, 391-394.

Pena, J. And Grace, J. (1986). Water relations and ultrasound emissions of Pinus sylvestris before, during and after a period of water stress. New Phytol. 103, 515-524.

Pickard, W. F. (1981). The ascent of sap in plants. Prog. Biophys. molec. Biol. 37, 181-229.

PICKARD, W. F. (1989). How might a tracheary element which is embolized by day be healed by night? J. theor. Biol. 141, 259-279.

Pickford, G. E. And McConnaughey, B. H. (1949). The Octopus bimaculatus problem: a study in sibling species. Bull. Bingham Oceanogr. Collect. Yale Univ. 12, 1-66.

Salleo, S. ANd LoGullo, M. A. (1986). Xylem cavitation in nodes and internodes of whole Chorisia insignis plants subjected to water stress: relations between xylem conduit size and cavitation. Ann. Bot. 58, 431-442.

Scholander, P. F., Hammel, H. T., Bradstreet, E. D. and Hemmingsen, E. A. (1965). Sap pressures in vascular plants. Science 148, 339-346.

Sedgewick, S. A. AND Trevena, D. H. (1976). Limiting negative pressure of water under dynamic stressing. J. Phys. D 9, 1983-1990.

Shafrin, E. G. (1975). Critical surface tensions of polymers. In Polymer Handbook, 2nd edn (ed. J. Brandrup and E. H. Immergut), pp. 221-228. New York: John Wiley and Sons.

SPERRY, J. S. (1986). Relationship of xylem embolism to xylem pressure potential, stomatal closure, and shoot morphology in the palm Rhapsis excelsa. Plant Physiol. 80, 110-116.

SPERRY, J. S. AND TYREE, M. T. (1988). Mechanism of water stress-induced xylem embolism. Plant Physiol. 88, 581-587.

STORK, N. E. (1980). Experimental analysis of adhesion of Chrysolina polita (Chrysomelidae: Coleoptera) on a variety of surfaces. J. exp. Biol. 88, 91-107.

Thomas, L. A. and Hermans, C. O. (1985). Adhesive interactions between the tube feet of a 
starfish, Leptasterias hexactis, and substrata. Biol. Bull. mar. biol. Lab., Woods Hole 169, $675-688$.

Trevena, D. H. (1967). The behavior of liquids under tension. Contemp. Phys. 8, 185-195.

TyreE, M. T. AND Dixon, M. A. (1986). Water stress induces cavitation and embolism in some woody plants. Physiol. Plant. 66, 397-405.

VolGht, J. R. (1990). Population biology of Octopus digueti and the morphology of American tropical octopods. PhD dissertation, University of Arizona, Tucson.

Wilson, D. A., HoYt, J. W. ANd McKune, J. W. (1975). Measurement of tensile strength of liquids by an explosion technique. Nature 253, 723-725.

Yule, A. B. AND CRisp, D. J. (1983). Adhesion of cypris larvae of the barnacle, Balanus balanoides, to clean and arthropodin treated surfaces. J. mar. biol. Ass. U.K. 63, 261-271.

Zimmerman, M. H. (1983). Xylem Structure and the Ascent of Sap. Berlin: Springer-Verlag. 
